\title{
Boas práticas na assistência ao parto natural
}

\author{
Best practices in natural childbirth care \\ Buenas prácticas en la atención al parto natural
}

Recebido: 23/04/2021 | Revisado: 02/05/2021 | Aceito: 02/05/2021 | Publicado: 15/05/2021

\author{
Roberto de Sousa Costa \\ ORCID: https://orcid.org/0000-0003-0285-8409 \\ Centro Universitário Santo Agostinho, Brasil \\ E-mail:robertocosta.enf@gmail.com \\ Janaína Pereira Ferreira \\ ORCID: https://orcid.org/0000-0003-4844-4981 \\ Centro Universitário Santo Agostinho, Brasil \\ E-mail: ferreinhanet987@gmail.com \\ Magda Rogéria Pereira Viana \\ ORCID: https://orcid.org/0000-0003-3293-7095 \\ Centro Universitário Santo Agostinho, Brasil \\ E-mail: magdarogeria@ hotmail.com
}

\begin{abstract}
Resumo
O desenvolvimento de boas práticas na assistência ao parto normal, implementadas nas rotinas dos centros obstétricos, é importante, considerando-se a humanização do binômio materno-fetal, que favorece uma experiência positiva, bem como, contribui para o processo de parturição de forma que a mãe se sinta acolhida e possa manter saúde física e emocional, garantindo um parto sem complicações e que lhe traga boas recordações desse momento. O objetivo desse trabalho foi avaliar quais os conhecimentos sobre as boas práticas adotadas por enfermeiros que atuam na assistência ao parto natural, conforme as publicações científicas. Utilizou-se a revisão integrativa, em que 11 artigos científicos disponibilizados na íntegra, nos últimos 5 anos, selecionados na Biblioteca Virtual de Saúde, compuseram a amostra analisada. Os resultados demonstraram que, em todas as publicações foram empregadas boas práticas assistenciais, principalmente as não farmacológicas, por enfermeiros, para alívio da dor, oportunizando um trabalho de parto fisiológico e humanizado. Conclui-se que as estratégias empregadas pelos enfermeiros contribuem para o resgate do parto natural, que apresenta inúmeras vantagens para o binômio mãe e filho.
\end{abstract}

Palavras-chave: Gravidez; Cuidados de enfermagem; Parto normal; Guia de prática clínica.

\begin{abstract}
The development of good practices in assisting normal childbirth, implemented in the routines of obstetric centers, is important, considering the humanization of the maternal-fetal binomial, which favors a positive experience, as well as contributing to the parturition process so that the mother feels welcomed and can maintain physical and emotional health, ensuring an uncomplicated delivery and that brings good memories of that moment. The objective of this work was to evaluate what knowledge about the good practices adopted by nurses who work in assisting natural childbirth, according to scientific publications. The integrative research method was used, in which 11 scientific articles made available in full, in the last 5 years, selected in the Virtual Health Library (VHL), comprised the analyzed sample. The results showed that in all publications good care practices were used, especially non-pharmacological ones by nurses for pain relief and providing physiological and humanized labor. We conclude that the strategies employed by nurses contribute to the rescue of natural childbirth, which has numerous advantages for the mother and child binomial.
\end{abstract}

Keywords: Pregnancy; Nursing care; Normal birth; Practice guideline.

\section{Resumen}

Es importante el desarrollo de buenas prácticas en la atención al parto normal, implementadas en las rutinas de los centros obstétricos, considerando la humanización del binomio materno-fetal, que favorece una experiencia positiva, además de contribuir al proceso del parto para que la madre se sienta bienvenidos y puedan mantener la salud física y emocional, garantizando un parto sin complicaciones y que traiga buenos recuerdos de ese momento. El objetivo de este trabajo fue evaluar qué conocimientos sobre las buenas prácticas adoptados por los enfermeros que laboran en la asistencia natural al parto, según publicaciones científicas. Se utilizó la revisión integradora, en la que 11 artículos científicos puestos a disposición en su totalidad, en los últimos 5 años, seleccionados en la Biblioteca Virtual en Salud, conformaron la muestra analizada. Los resultados mostraron que, en todas las publicaciones, las enfermeras utilizaron buenas prácticas asistenciales, principalmente no farmacológicas, para el alivio del dolor, proporcionando un trabajo fisiológico y humanizado. Se concluye que las estrategias empleadas por las enfermeras contribuyen a rescatar el parto natural, que tiene numerosas ventajas para el binomio madre e hijo. 
Palabras clave: Embarazo; Cuidado de enfermeira; Parto normal; Guía de práctica clínica.

\section{Introdução}

A atenção ao parto e ao nascimento vem passando por diversas mudanças nos últimos anos, nos âmbitos nacionais e internacionais. O nascer é um fato e um acontecimento natural. Essa realização representa um fenômeno marcante para a mãe e toda sua família (Pereira et al., 2018).

No Brasil cerca de três milhões de crianças nascem por ano, seja em hospitais públicos ou privados, fato que acaba envolvendo praticamente um número dobrado de pessoas nesse processo, vez que a gestante espera a tão sonhada chegada do filho (a) ao mundo, momento que ficará sempre marcado na sua vida e envolve toda uma equipe, na qual é responsável pela assistência à parturiente e a toda à família (Brasil, 2017).

Para Vendrúscolo e Kruel (2015), a assistência ao parto passou por diversas transformações evolutivas no decorrer dos tempos, das residências familiares aos estabelecimentos de saúde, de um evento que envolvia as conhecidas parteiras a um evento mais técnico-profissional, na pessoa dos médicos obstetras, da não-medicalização a medicalização, do natural a um evento mais técnico ou artificial.

Foram tantas as transformações nesse campo, desenvolvidas para melhor e de forma mais eficiente, para atender a equipe de saúde e a gestante, que a parturiente passou de sujeito a objeto, de sujeito ativo a sujeito passivo, ou seja, uma pessoa que pouco ou nada participa e/ou decide a respeito de como o parto será conduzido. Em razão disso, existe um movimento de humanização do parto, preconizado pelo Ministério da Saúde (MS), solicitando uma assistência integral e humanizada à mulher, como uma tentativa de empoderamento da mulher neste momento tão crucial e único em sua vida, conforme descrevem Nagahama e Santiago (2005).

Com as mudanças que inevitavelmente ocorreram na história, mudou-se também a assistência e práticas utilizadas no parto, composta de políticas e diretrizes que norteiam e preconizam a assistência com um enfoque de cuidado e humanização, desde o pré-natal, parto, e até o pós-parto, garantindo à mulher todos seus direitos, que ficará marcado na sua vida e proporcionando à mãe, as melhores experiências possíveis em relação às práticas adotadas no momento do parto (Brasil, 2017).

Nessa perspectiva, o desenvolvimento de boas práticas na assistência ao parto normal, implementadas nas rotinas dos centros obstétricos, é importante, considerando-se a humanização do binômio materno-fetal, que favorece uma experiência positiva, bem como, contribui para o processo de parturição de forma que a mãe se sinta acolhida e possa manter saúde física e emocional, garantindo um parto sem complicações e que lhe traga boas recordações desse momento (Carvalho \& Silva, 2020).

Porém, outras práticas, como a episiotomia de rotina, aspiração naso-faríngeana o uso de ocitocina, a cesariana, dentre outras, são utilizadas em diferentes ambientes de assistência ao parto, em instituições públicas ou privadas, que de forma frequente, o que coloca em risco a segurança das parturientes e de seus filhos, porque nem sempre são as melhores escolhas no momento do processo do parto, ou seja, deveriam ser utilizadas de forma parcimoniosa e não como métodos rotineiros como são reportados em muitos estudos (Brasil, 2017).

O Ministério da Saúde (MS) evidencia a existência de altas taxas de traumas durante o parto, tanto para o bebê quanto para a mãe. O conhecimento do enfermeiro obstetra no campo é fundamental para o processo fisiológico de parturição, o que melhora os resultados, quando comparado com tempos mais antigos, em que não existiam profissionais especializados/capacitados no processo de partejar. Com a prática efetiva e reiterada desse conhecimento por parte desse profissional, ousamos afirmar que isso ensejaria uma diminuição de forma gradativa de tais sinistros que atualmente culminam, às vezes, em letalidade (Brasil, 2017). 
A Organização Mundial da Saúde (OMS), desenvolveu uma classificação das práticas comuns na condução do parto normal, orientando o que deve ser feito no processo do parto, que foi dividida em quatro categorias: I) práticas úteis e que devem ser estimuladas; (II) práticas prejudiciais ou que não apresentam nenhuma eficácia na sua aplicação; (III) práticas sem evidências que possam comprová-las e que devem ser colocadas em prática com mais cautela até que se tenham comprovações da sua eficácia e (IV) práticas realizadas inadequadamente pelos profissionais.

O MS tem criado cada vez mais políticas e diretrizes a favor da humanização e aplicação das boas práticas, no âmbito do parto e nascimento, para diminuir os riscos de problemas e trazer uma assistência obstétrica de qualidade, com uma atenção humanizada para o processo fisiológico de parturição, o que tem trazido melhores resultados, quando comparado com tempos mais antigos, em que não existiam profissionais especializados/capacitados no processo de partejar. Os profissionais que atuam nessa área, em destaque o Enfermeiro obstetra, têm uma aliada patente de informações que lhe asseguram quais decisões poderão ser tomadas de acordo com o momento do parto (Brasil, 2017).

De maneira geral, o parto normal, proporciona uma série de vantagens e é um evento natural, que tem menor custo, também é um importante complemento na maturidade do recém-nascido, que, ao passar pela bacia da mãe, o bebê tem seu tórax comprimido, facilitando-lhe a respiração e diminuindo o risco de problemas respiratórios, por expelir líquido que, porventura, possa estar depositado em seus pulmões (Brasil, 2013).

Em virtude dos fatos mencionados, toda mulher tem o direito de escolher que tipo de parto ela deseja ser submetida, e também a aplicabilidade das boas práticas, como por exemplo, Informações e comunicação; Apoio físico e emocional; Dieta durante o trabalho de parto; Medidas de assepsia para o parto vaginal; Avaliação do bem-estar fetal; Estratégias e métodos não farmacológicos de alívio da dor no trabalho de parto, dentre outras práticas, uma vez que o parto natural é o mais indicado por apresentar uma série de vantagens que lhe proporcionam um bem-estar mais rápido (Brasil, 2017).

A criação de um vínculo entre o Enfermeiro obstetra e a parturiente, com explicação do que será realizado em relação às boas práticas no momento do parto resulta em um atendimento mais humanizado e proporciona melhores resultados para o binômio materno-fetal. Dessa forma, objetiva-se com a investigação descrever as boas práticas na assistência ao parto natural, evidenciadas nas publicações científicas sobre a temática.

\section{Metodologia}

Trata-se de uma revisão da literatura integrativa, que de acordo com Gil (2017), é uma pesquisa realizada com base em material já publicado. Tradicionalmente, esta modalidade de pesquisa inclui material impresso. Todavia, em virtude da disseminação de novos formatos de informação, estas pesquisas passaram a incluir outros tipos de fontes, como o material disponibilizado pela Internet onde se buscam todos os materiais já publicados em relação ao tema requisitado, principalmente artigos científicos originais.

Para a busca de dados, utilizou-se como fonte de coleta, a plataforma online de pesquisa Biblioteca Virtual em Saúde (BVS) nas bases de dados Medical Literature Analysis and Retrieval System Online (MEDLINE), Literatura Latino Americana e do Caribe em Ciências da Saúde (LILACS), Base de dados em Enfermagem (BDENF) e web artigos, segundo os critérios de inclusão. Para a elaboração da questão de pesquisa da revisão integrativa, utilizou-se a estratégia PICo, fundamental para a melhora da especificidade e clareza conceitual da situação clínica a ser estudada retornando resultados de maior qualidade e precisão, maximizando assim as informações relevantes a serem utilizadas no estudo para atender aos objetivos propostos e evitando informações desnecessárias (Landa-Ramirez \& Arredondo-Pantalleón, 2014).

O grupamento "P" refere-se à população do estudo (gravidez); "I" à intervenção estudada ou à variável de interesse (cuidados de enfermagem); "Co" ao contexto de análise da pesquisa (parto natural), a busca dos artigos foi realizada 
utilizando-se os Descritores em Ciências da Saúdes (DeCS) e Medical Subject Headings (MeSH) a partir dos operadores booleanos "AND" e "OR", como descrito no Quadro 1.

Quadro 1. Apresentação da estratégia PICo para elaboração da pergunta norteadora e estratégia de busca.

\begin{tabular}{|l|l|l|}
\hline Acrônimos & Componentes & Descs/Machs \\
\hline P & População & Gravidez, pregnancy, embarazo \\
\hline I & Fenômenos de interesse & "Cuidados de Enfermagem", "Nursing Care", "Atención de Enfermería" \\
\hline Co & Contexto & "Parto Normal", "Natural Childbirth", "Parto Normal" \\
\hline
\end{tabular}

Fonte: Biblioteca Virtual de Saúde (BVS).

O uso e combinação dos descritores da estratégia PICo é fundamental para obtenção de um maior número de resultados, atendendo aos objetivos deste estudo, que no caso deste estudo resultou na seguinte questão: "Quais as boas práticas na assistência ao parto natural evidenciadas nas publicações científicas?"

Como critério de inclusão foram selecionados os artigos indexados nos últimos 5 anos, em periódicos nacionais e internacionais, disponibilizados na íntegra, em língua portuguesa, inglesa e espanhola que corresponderam à temática em estudo. Excluíram-se do estudo, publicações cujos títulos e/ou objetivos não possuíam ligação direta com a temática e o objetivo do estudo, textos incompletos (resumos), teses e/ou monografias e artigos duplicados, conforme mostra a Figura 1.

Figura 1. Fluxograma com resultados das buscas nas bases de dados ( $\mathrm{n}=11)$.

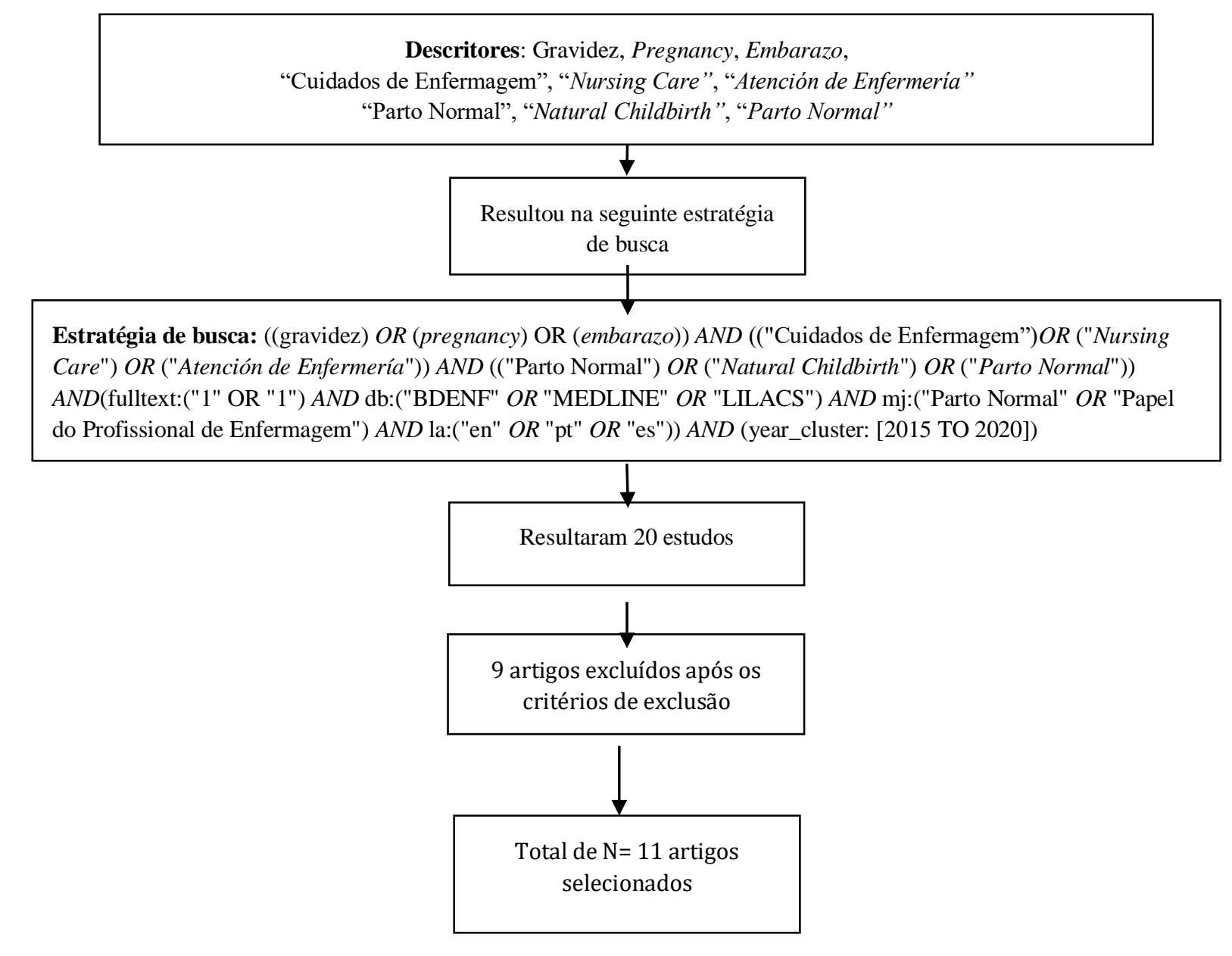

Fonte: Biblioteca Virtual de Saúde. 
Para a busca dos artigos foi utilizado um formulário semiestruturado, que contemplou dois eixos de informações: no Eixo 1- Perfil das produções científicas: Título, ano, autores, idioma, base de dados e abordagem metodológica; Eixo 2Resultados em evidência, com o seguinte questionamento: Quais as boas práticas na assistência ao parto natural evidenciadas na publicação?

Os produtos da revisão foram representados através de quadros, utilizando a ferramenta Microsoft Word, conforme os tópicos estabelecidos no formulário. A análise dos estudos foi feita a partir da similaridade semântica, para posterior análise, conforme os artigos pesquisados e discutidos com base dos estudos resultantes da busca.

\section{Resultados}

A partir da leitura e análise do material encontrado conforme os critérios de elegibilidade, foram agrupadas informações pertinentes sobre os artigos encontrados, e os dados obtidos foram divididos em dois quadros. O Quadro 2 representando o perfil das produções, no qual abordou-se os seguintes itens: Título, ano, autores, idioma, abordagem e base de dados.

Quadro 2. Eixo 1- Perfil das produções (n=11).

\begin{tabular}{|c|c|c|c|c|c|c|}
\hline $\mathbf{N}^{\mathbf{o}}$ & Título & Ano & Autores & Idioma & Abordagem & $\begin{array}{c}\text { Base de } \\
\text { dados }\end{array}$ \\
\hline 01 & $\begin{array}{c}\text { Práticas de enfermeiras } \\
\text { para promoção da } \\
\text { dignificação, participação } \\
\text { e autonomia de mulheres } \\
\text { no parto normal }\end{array}$ & 2015 & SILVA et al & Português & Qualitativo & LILACS \\
\hline 02 & $\begin{array}{c}\text { Perfil da assistência } \\
\text { hospitalar prestada por } \\
\text { enfermeiras residentes em } \\
\text { obstetrícia em Brasília, } \\
\text { Distrito Federal }\end{array}$ & 2016 & $\begin{array}{c}\text { CAMPOS et } \\
a l\end{array}$ & Português & Quantitativo & LILACS \\
\hline 03 & $\begin{array}{c}\text { The Value of the } \\
\text { Maternity Care Team } \\
\text { in the Promotion of } \\
\text { Physiologic Birth }\end{array}$ & 2016 & $\begin{array}{l}\text { ZIELINSKI et } \\
\text { al. }\end{array}$ & Inglês & Qualitativo & MEDLINE \\
\hline 04 & $\begin{array}{c}\text { Importance of the Birth } \\
\text { Environment to } \\
\text { Support Physiologic Birth }\end{array}$ & 2016 & STARK et al. & Inglês & Qualitativo & MEDLINE \\
\hline 05 & $\begin{array}{l}\text { SATISFAÇÃO DAS } \\
\text { PUÉRPERAS } \\
\text { ATENDIDAS EM UM } \\
\text { CENTRO DE PARTO } \\
\text { NORMAL }\end{array}$ & 2017 & $\begin{array}{l}\text { SOARES et } \\
\text { al. }\end{array}$ & Português & Qualitativa & BDENF \\
\hline 06 & $\begin{array}{l}\text { TECNOLOGIAS NÃO } \\
\text { INVASIVAS DE } \\
\text { CUIDADO: } \\
\text { PERCEPÇÃO DAS } \\
\text { PUÉRPERAS }\end{array}$ & 2018 & $\begin{array}{l}\text { PEREIRA et } \\
\text { al. }\end{array}$ & Português & Qualitativo & BDENF \\
\hline 07 & $\begin{array}{c}\text { A HUMANIZAÇÃO NA } \\
\text { ASSISTÊNCIA AO } \\
\text { PARTO E AO } \\
\text { NASCIMENTO }\end{array}$ & 2018 & $\begin{array}{l}\text { CORDEIRO } \\
\text { et al. }\end{array}$ & Português & Quantitativo & BDENF \\
\hline 08 & $\begin{array}{c}\text { INTERDISCIPLINARITY } \\
\text { IN CHILDBIRTH CARE: } \\
\text { PERCEPTION OF } \\
\text { OBSTETRIC NURSES }\end{array}$ & 2019 & BRAZ et al. & Inglês & Qualitativo & BDENF \\
\hline
\end{tabular}




\begin{tabular}{|c|c|c|c|c|c|c|}
\hline 09 & $\begin{array}{c}\text { Comunicação não verbal } \\
\text { enfermeiro-parturiente no } \\
\text { trabalho de parto } \\
\text { em países lusófonos }\end{array}$ & 2019 & $\begin{array}{c}\text { BESERRA } \text { et } \\
\text { al. }\end{array}$ & Português & Quantitativo & LILACS \\
\hline 10 & $\begin{array}{c}\text { Partejar de primíparas: } \\
\text { reflexos na amamentação }\end{array}$ & 2019 & $\begin{array}{c}\text { CASTRO } \text { et } \\
\text { al }\end{array}$ & Português & Qualitativo & LILACS \\
\hline $\begin{array}{c}\text { TECNOLOGIAS DE } \\
\text { CUIDADO PARA O } \\
\text { ALÍVIO DA DOR NA } \\
\text { PARTURIÇÃO }\end{array}$ & 2020 & $\begin{array}{c}\text { MARINS } \text { et } \\
\text { Pl }\end{array}$ & Português & Qualitativo & LILACS \\
\hline
\end{tabular}

Fonte: Biblioteca Virtual de Saúde (BVS).

Como demonstra a figura anterior, observa-se grande distribuição entre os anos de publicação, no entanto, o ano de 2016 e 2019 apresentam maior frequência de publicação de artigos acerca da temática totalizando 3 publicações em ambos os anos. Com relação ao idioma empregado não foram encontrados estudos na língua espanhola, sendo a língua portuguesa a mais presente, empregada em mais da metade das amostras, mais precisamente em 8 publicações. Na abordagem metodológica são 8 estudos qualitativos e apenas 3 quantitativos. Nas publicações, vale destacar a diversidade de periódicos, em que houve o emprego maior na base de dados Literatura Latino Americana e do Caribe em Ciências da Saúde (LILACS), com 5 publicações, em seguida na Base de dados em Enfermagem (BDENF), com 4 publicações e por fim na Medical Literature Analysis and Retrieval System Online (MEDLINE), com as 2 publicações restantes.

Já o Quadro 3 aborda os resultados em evidência retirados de cada publicação após uma análise criteriosa, feita por dois revisores separadamente, e enviadas a um terceiro, a fim de responder o seguinte questionamento: Quais as boas práticas na assistência ao parto natural evidenciadas na publicação?

Quadro 3. Resultados em evidência ( $\mathrm{n}=11)$.

\begin{tabular}{|c|c|c|}
\hline $\mathbf{N}^{\mathbf{o}}$ & Título & $\begin{array}{c}\text { Quais as boas práticas na assistência ao parto natural evidenciadas na } \\
\text { publicação? }\end{array}$ \\
\hline 01 & $\begin{array}{l}\text { Práticas de enfermeiras para } \\
\text { promoção da dignificação, } \\
\text { participação e autonomia de } \\
\text { mulheres no parto normal }\end{array}$ & $\begin{array}{l}\text { O estudo apontou que as práticas de cuidado utilizadas por enfermeiras } \\
\text { obstétricas de um CPN, implicadas nos processos de dignificação foram o } \\
\text { acolhimento; a promoção da presença de acompanhante; a promoção de um } \\
\text { ambiente adequado ao cuidado; e a transmissão de calma e segurança às } \\
\text { mulheres. Já a autonomia das mulheres no trabalho de parto foi possível por } \\
\text { meio da promoção de relações pessoais entre profissionais e usuárias livres de } \\
\text { coerção; e a facilitação no acesso às informações. }\end{array}$ \\
\hline 02 & $\begin{array}{l}\text { Perfil da assistência hospitalar } \\
\text { prestada por enfermeiras } \\
\text { residentes em obstetrícia em } \\
\text { Brasília, Distrito Federal }\end{array}$ & $\begin{array}{l}\text { Na maioria dos partos assistidos pelas enfermeiras residentes em obstetrícia não } \\
\text { houve a utilização de ocitocina nem a realização da episiotomia. Na condução do } \\
\text { parto, os exercícios facilitadores mais utilizados foram as posições verticais e a } \\
\text { deambulação. As enfermeiras residentes em obstetrícia, predominantemente, } \\
\text { estabelecem práticas de cuidado humanizado baseadas em evidências científicas. }\end{array}$ \\
\hline 03 & $\begin{array}{c}\text { The Value of the Maternity Care } \\
\text { Team in the Promotion of } \\
\text { Physiologic Birth }\end{array}$ & $\begin{array}{l}\text { O estudo apontou que os benefícios do parto normal e fisiológico foram bem } \\
\text { documentados. Profissionais de saúde, como enfermeiras, parteiras,e os médicos } \\
\text { têm papéis distintos e significativos na promoção dos processos fisiológicos do } \\
\text { nascimento. A equipe multiprofissional deverá manter um diálogo, afim de um } \\
\text { único objetivo: Facilitar o processo de parto normal e tornar uma experiência } \\
\text { mais humana e satisfatória }\end{array}$ \\
\hline 04 & Importance of the Birth & O estudo apontou que o ambiente em que o parto irá acontecer interfere bastante \\
\hline
\end{tabular}




\begin{tabular}{|c|c|c|}
\hline & $\begin{array}{c}\text { Environment to Support } \\
\text { Physiologic Birth }\end{array}$ & $\begin{array}{l}\text { de modo positivo ou negativo, dependendo das condições abordadas, para o } \\
\text { processo de humanização e alívio da dor. Além disso, as enfermeiras que têm } \\
\text { contato pré-natal com mulheres grávidas e seus as famílias podem fornecer } \\
\text { informações sobre locais de nascimento e apoiar as mulheres em suas decisões. }\end{array}$ \\
\hline 05 & $\begin{array}{l}\text { SATISFAÇÃO DAS } \\
\text { PUÉRPERAS ATENDIDAS } \\
\text { EM UM CENTRO DE PARTO } \\
\text { NORMAL }\end{array}$ & $\begin{array}{l}\text { Neste estudo foi possível constatar que as puérperas se mostraram satisfeitas em } \\
\text { relação ao atendimento e estrutura oferecida pelo CPN, uma vez que esse serviço } \\
\text { ofereceu à parturiente vínculo com acompanhante, acolhimento, ambiente } \\
\text { confortável e silencioso, técnicas não farmacológicas como: banho quente, } \\
\text { exercícios perineais com bola suíça, exercícios de respiração, relaxamento, } \\
\text { massagem, acupuntura, movimentos corporais entre outros e equipe } \\
\text { multiprofissional preparada. }\end{array}$ \\
\hline 06 & $\begin{array}{l}\text { TECNOLOGIAS NÃO } \\
\text { INVASIVAS DE CUIDADO: } \\
\text { PERCEPÇÃO DAS } \\
\text { PUÉRPERAS }\end{array}$ & $\begin{array}{l}\text { O estudo mostrou que a assistência prestada pelos profissionais, principalmente } \\
\text { os de Enfermagem, do centro obstétrico, foi percebida pelas puérperas como } \\
\text { cuidados que aliviaram a dor e proporcionaram bem-estar, cuidados que } \\
\text { aceleraram o trabalho de parto, orientações por parte dos profissionais e cuidados } \\
\text { que contribuíram, de alguma maneira, durante a sua internação. }\end{array}$ \\
\hline 07 & $\begin{array}{c}\text { A HUMANIZAÇÃO NA } \\
\text { ASSISTÊNCIA AO PARTO E } \\
\text { AO NASCIMENTO }\end{array}$ & $\begin{array}{l}\text { O estudo apontou que os enfermeiros utilizaram técnicas de relaxamento para a } \\
\text { promoção do alívio da dor e exercícios facilitadores do trabalho de parto, o } \\
\text { esclarecimento à parturiente sobre o seu direito a um acompanhante, ofereceram } \\
\text { liberdade para que houvesse a escolha da posição de parto, a deambulação e a } \\
\text { oferta de líquidos claros e alimentação que contribuiu positivamente para } \\
\text { assistência à mãe e ao seu concepto. }\end{array}$ \\
\hline 08 & $\begin{array}{l}\text { INTERDISCIPLINARITY IN } \\
\text { CHILDBIRTH CARE: } \\
\text { PERCEPTION OF OBSTETRIC } \\
\text { NURSES }\end{array}$ & $\begin{array}{l}\text { Revela-se que as enfermeiras obstétricas demonstraram ter conquistado um } \\
\text { espaço significativo para o seu trabalho, possibilitando-lhes respeitar o processo } \\
\text { natural do parto e a mulher como protagonista, oferecendo-lhe o suporte e a } \\
\text { segurança necessários para realizar o cuidado. }\end{array}$ \\
\hline 09 & $\begin{array}{l}\text { Comunicação não verbal } \\
\text { enfermeiro-parturiente no } \\
\text { trabalho de parto em países } \\
\text { lusófonos }\end{array}$ & $\begin{array}{l}\text { Conforme se concluiu, enxerga-se, neste estudo, a relevância da comunicação } \\
\text { não verbal no processo de cuidar no cenário obstétrico nos dois países, com } \\
\text { vistas a proporcionar atendimento humanizado e de qualidade. A enfermagem foi } \\
\text { fundamental para o repasse de informações e conduta que facilitasse a } \\
\text { comunicação não verbal entre profissionais e paciente. }\end{array}$ \\
\hline 10 & $\begin{array}{c}\text { Partejar de primíparas: reflexos } \\
\text { na amamentação }\end{array}$ & $\begin{array}{l}\text { Foi possível identificar que o processo de amamentar no CPN ocorreu de } \\
\text { maneira satisfatória para a maioria das mães, sendo uma experiência positiva, } \\
\text { prazerosa e de facilidade na prática. Evidenciou-se que o parto em CPN contribui } \\
\text { para que essa prática aconteça de maneira natural e de forma imediata, uma vez } \\
\text { que esse espaço proporciona proximidade entre mãe e bebê e fortalecimento do } \\
\text { vínculo. }\end{array}$ \\
\hline 11 & $\begin{array}{c}\text { TECNOLOGIAS DE } \\
\text { CUIDADO PARA O ALÍVIO } \\
\text { DA DOR NA PARTURIÇÃO }\end{array}$ & $\begin{array}{l}\text { O estudo revelou que as tecnologias não farmacológicas devem ser utilizadas } \\
\text { com o intuito de promover assistência humanizada, sem o uso da medicalização } \\
\text { e procedimentos invasivos desnecessários. É imperativa a necessidade do uso } \\
\text { dessas ações assistenciais para diminuir o nível de estresse e ansiedade da mulher } \\
\text { durante o trabalho de parto, pois, mesmo utilizando os analgésicos, isolados eles } \\
\text { não resolvem totalmente o fenômeno multidimensional que é a dor. }\end{array}$ \\
\hline
\end{tabular}


Analisou-se que todos os 11 artigos trabalhavam assiduamente a importância das boas práticas na assistência ao parto natural, que contribuem para o processo de humanização, tornam esse momento tão importante para a mãe e a família ainda mais inesquecível, e fortalece vínculos. Além disso, foi possível destacar o papel da enfermagem no incremento dessas práticas e no cuidado mais empático com as parturientes.

\section{Discussão}

Silva, Nascimento e Coelho (2015), realizaram um estudo levando em consideração as experiências vividas por mulheres que fizeram o seu parto de maneira natural, atendidas nos Centros de Parto Natural (CPN). A pesquisa demonstrou que as práticas de cuidado utilizadas por enfermeiras obstétricas, implicadas nos processos de dignificação foram o acolhimento; a promoção da presença de acompanhante; a promoção de um ambiente adequado ao cuidado; e a transmissão de calma e segurança às mulheres. Já a autonomia das mulheres no trabalho de parto foi possível por meio da promoção de relações pessoais entre profissionais e usuárias livres de coerção; e a facilitação no acesso às informações. O suporte fornecido por enfermeiras durante o trabalho de parto dignificou o cuidado de enfermagem e evidenciou que a sua atuação em CPN deve ser valorizada, visto que nesse local elas têm mais autonomia para atuarem.

Em outro estudo realizado, Soares et al (2017), evidenciou a satisfação das puérperas que também foram atendidas nos CNP. Os resultados foram positivos ao passo que foram utilizadas tecnologias não invasivas para alívio da dor como por exemplo: banho quente, exercícios perineais com bola suíça, exercícios de respiração, relaxamento, massagem, acupuntura. Estas práticas oferecem conforto e possibilitam a liberdade de movimento das parturientes. Além disso, as mulheres foram assistidas em um ambiente silencioso, harmônico e confortável, e foi estimulado a presença do acompanhante. Destaca-se o papel da equipe de enfermagem que contribuiu para a construção de uma relação empática o que favoreceu a satisfação, pois as puérperas se sentiram acolhidas e amparadas, acarretando maior confiança e segurança no trabalho de parto e parto, resultando em conforto, além de favorecer o protagonismo da mulher diante do processo parto-nascimento.

Em concordância a pesquisa realizada por Castro et al (2019), também aborda satisfação das mulheres que foram atendidas no CNP, e que isso refletiu na prática da amamentação. Foi possível identificar que o processo de amamentar no CPN ocorreu de maneira satisfatória para a maioria das mães, sendo uma experiência positiva, prazerosa e de facilidade na prática. Evidenciou-se que o parto em CPN contribui para que essa prática aconteça de maneira natural e de forma imediata, uma vez que esse espaço proporciona proximidade entre mãe e bebê e fortalecimento do vínculo.

Em outra abordagem Pereira P. et al (2018), destacam em sua pesquisa o uso de tecnologias não invasivas de cuidado (TCNIC) para alívio da dor durante o período de parto. Percebeu-se, no estudo realizado, que a maioria das puérperas entrevistadas teve a oportunidade de receber cuidados relativos à massagem e/ou banho como medidas de alívio da dor, além de proporcionar conforto e bem-estar a essas pacientes. Ressaltou-se ainda que, entre as manifestações quanto ao uso das TCNIC no intuito de que as mesmas acelerassem o trabalho de parto de forma segura, tornando menor o tempo de espera para o parto, além de tornar a paciente protagonista desse processo parturitivo.

A pesquisa desenvolvida por Marins et al (2020), também destacou o uso de novas tecnologias que contribuem para facilitação do processo de parto e alívio da dor. O estudo aponta que as tecnologias não farmacológicas são utilizadas com o intuito de promover assistência humanizada, sem o uso da medicalização e procedimentos invasivos desnecessários. Medidas para aumentar o conforto e reduzir a apreensão durante todas as fases do trabalho de parto devem ter seu início durante a gestação, por meio da educação e aconselhamento durante o pré-natal, para que as mulheres sejam capazes de fazer escolhas. Outras estratégias que podem ser utilizadas são: a técnica de movimentação da bola, balançar ou fazer outros movimentos rítmicos podem ser reconfortantes por oportunizar o relaxamento da musculatura pélvica. Posições ortostáticas, como inclinar 
o corpo para frente ou usar a bola de parto como apoio durante as contrações conferem à maioria das mulheres uma sensação maior de controle e de movimento ativo do que apenas o ato de permanecer deitada.

O artigo de Cordeiro et al (2018), enfatizou que para proporcionar uma atenção humanizada, os profissionais de saúde deverão fornecer informações adequadas para cada situação, do pré-natal ao puerpério, oferecendo apoio físico e emocional, informando sobre o direito ao acompanhante e acalmando suas angústias e medos. Incluem-se, nas práticas de humanização do parto a oferta de dieta e técnicas para o alívio da dor como: banho morno, massagens, musicoterapia, cavalinho, bola suíça, liberdade para a posição de parto, deambulação, ambiente aconchegante, dentre outros.

Beserra et al (2019), abordaram a comunicação não verbal como uma estratégia e boa prática para o processo de parto. O seu estudo descreveu que a mulher precisa sentir-se acolhida para se comunicar com iniciativa e liberdade com os enfermeiros que lhe assistem no trabalho de parto. Uma comunicação não verbal efetiva implica resultados válidos e positivos tanto nos aspectos físicos quanto psicológicos dessa mulher. Além disso, a enfermagem desempenha papel importante no estabelecimento das técnicas e vínculos que proporcionem essa comunicação com as pacientes, enxerga-se, a relevância da comunicação não verbal no processo de cuidar no cenário obstétrico, com vistas a proporcionar atendimento humanizado e de qualidade.

Além disso, o estudo de Stark, Remynse e Zwelling (2016), demonstrou que o ambiente em que o parto irá acontecer interfere bastante de modo positivo ou negativo, dependendo das condições abordadas, para o processo de humanização e alívio da dor. Estratégias como musicoterapia à escolha da mulher; vistas do ambiente natural por meio de uma janela, fotos ou DVDs; e redução de ruído e pessoal e conversas desnecessárias são maneiras que os enfermeiros podem criar um ambiente calmo. O comportamento das enfermeiras também apoia o parto fisiológico, quando estas permanecem calmas, e quando estabelecem uma linguagem de incentivo e defesa do nascimento fisiológico. Além disso, é possível inferir que os enfermeiros que têm contato desde o pré-natal com essas mulheres grávidas e seus familiares, podem fornecer informações sobre locais de nascimento e apoiá-las em suas decisões.

Em concordância ao apoio dos benefícios do parto fisiológico a pesquisa elaborada por Zielinski, Brody e Low (2016), ressaltou que os enfermeiros são as principais fontes de apoio e educação para mulheres e famílias durante a gravidez, trabalho de parto e nascimento. Quando enfermeiros e outros provedores orientam uma mulher de uma forma que honra seus sentimentos, a experiência do parto e nascimento é otimizado. Além disso, o parto fisiológico deve ser incentivado e desmistificado pelos profissionais, para que a mulher sinta-se confiante e respeitada. Práticas de parto fisiológicas baseadas em evidências melhoraram os resultados de saúde para mães e recém-nascidos, no entanto, eles não são consistentemente endossados ou praticados por todos os profissionais de maternidade. Por meio dos esforços de indivíduos, instituições, formuladores de políticas e organizações, as mudanças podem ocorrer que irá promover o nascimento baseado em evidências práticas que otimizam os resultados para as mães e recém-nascidos e apoiar as escolhas das mulheres em relação às suas experiências de nascimento.

Por fim, os estudos de Campos et al (2016) e Braz et al (2019), ressaltam que dentre as técnicas e boas práticas empregadas para humanizar o processo de parto, a enfermagem se destaca, não só por ser a profissão detentora do cuidar, mas também, por manter uma visão mais holística e empática dos pacientes. No primeiro estudo constatou-se que na maioria dos partos assistidos pelas enfermeiras residentes em obstetrícia não houve a utilização de ocitocina nem a realização da episiotomia. Na condução do parto, os exercícios facilitadores mais utilizados foram as posições verticais e a deambulação. As enfermeiras residentes em obstetrícia, predominantemente, estabelecem práticas de cuidado humanizado baseadas em evidências científicas. Já o segundo estudo revelou que os enfermeiros obstétricos demonstraram ter conquistado um espaço significativo para o seu trabalho, ao longo dos anos por tamanha importancia, possibilitando-lhes respeitar o processo natural do parto e a mulher como protagonista, oferecendo-lhe o suporte e a segurança necessários para realizar o cuidado. 


\section{Conclusão}

A bibliografia levantada demonstrou que existem diversas práticas que podem ser empregadas para amenizar a dor, reduzir a ansiedade e o medo que as mulheres sentem quando estão em trabalho de parto.

É possível inferir que muitos artigos analisados se referem aos Centros de Parto Natural (CNP) como uma alternativa para a efetivação desse novo modelo de atenção obstétrica, pois busca resgatar a prática do parto normal que oferece maior segurança à saúde da mulher e ao recém-nascido, ao passo que tem como vantagem rápida recuperação pós-parto, baixo risco de infecção, hemorragias e outras complicações.

Além disso, para o emprego de boas práticas na assistência ao parto natural é necessário que haja o apoio e o envolvimento técnico e cientifico de todos os profissionais envolvidos na assistência as gestantes e aos seus familiares. Ainda assim, é possível destacar que a enfermagem é a categoria profissional que mais aplica cuidados e estratégias que tornam esse momento importante na vida da mulher, mais humanizado e inesquecível. Essas técnicas são em sua maioria não farmacológicas com o emprego de musicoterapia, banho morno, massagens, cavalinho, bola suíça, liberdade para a posição de parto, deambulação, ambiente aconchegante, estabelecimento de comunicação verbal e não verbal, estimulo a presença do acompanhante, e apoio as decisões e necessidades da gestante.

Diante de tal importância evidenciada pelo emprego do estimulo ao parto natural e as boas práticas que aliviam a dor e tornam o processo humanizado, sugere-se que haja a ampliação dos estudos que trabalhem a temática, para que o debate sobre o resgate do parto fisiológico, que por sua vez, reduz os riscos à saúde da mulher e do recém-nascido, estabelece o vínculo entre o binômio mãe e filho, torna a mulher protagonista de um dos momentos mais importantes da sua vida, e reduz as intervenções médicas desnecessárias, seja cada vez mais fortalecido afim de substituir o modelo hegemônico ainda empregado por muitos profissionais, por uma assistência holística e empática.

\section{Referências}

Beserra, G. de L., Oliveira, P. M. P. de, Pagliuca, L. M. F, Almeida, P. C. de, Anjos, S. de J. S. B. dos, \& Pinheiro, A. K. B. (2019). Comunicação não verbal enfermeira-parturiente em trabalho de parto em países de língua portuguesa. Revista Latino-Americana de Enfermagem, 27. https://doi.org/10.1590/15188345.3032 .3193

Brasil, \& Ministério da Saúde. (2013). Atenção ao pré-natal de baixo risco. Cadernos de Atenção Básica, n. 32.

Brasil. (2017). Ministério da saúde. Secretaria de Ciência, Tecnologia e Insumos Estratégicos. Departamento de Gestão e Incorporação de Tecnologias em Saúde. Diretrizes nacionais de assistência ao parto normal: versão resumida [recurso eletrônico] / Ministério da Saúde, Secre taria de Ciência, Tecnologia e Insumos Estratégicos, Departamento de Gestão e Incorporação de Tecnologias em Saúde. - Brasília: Ministério da Saúde

Braz, A. I. M., Gomes Paiva, M. T., Alves Feitosa, K. M., Soares Mendes, M. E., Alves Feitosa, T. M., \& Lins da Silva, S. (2019). Interdisciplinarity In Childbirth Care: Perception Of Obstetric Nurses. Journal of Nursing UFPE/Revista de Enfermagem UFPE, 13.

Campos, B. C. V., Pereira, É. P., Medeiros, G. A. D., \& Pereira, E. P. (2016). Perfil da assistência hospitalar por enfermeiras residentes em obstetrícia em Brasília, Distrito Federal. Comun. ciênc. saúde, 291-300.

Carvalho, S. S., \& da Silva, C. (2020). Boas práticas de enfermagem na assistência ao parto normal: revisão de literatura. Revista de Atenção à Saúde, 18(63).

Castro, I. R., de Melo, M. C. P., de Morais, R. J. L., \& dos Santos, A. D. B. (2019). Partejar de primíparas: reflexos na amamentação [Childbirth care for primiparas: reflexes in breastfeeding][Cuidado al parto de primiparas: reflejos en la lactancia materna]. Revista Enfermagem UERJ, $27,43354$.

Cordeiro, E. L., Silva, T. M. D., Silva, L. S. R. D., Veloso, A. C. F., Pimentel, R. V. T., Cabral, M. M. D. O., \& Silva, C. M. D. (2018). A humanização na assistência ao parto e ao nascimento. Rev. enferm. UFPE on line, 2154-2162.

Gil, A. C. (2017). Pós-Graduação-Metodologia-Como Elaborar Projetos de Pesquisa-Cap 2.

Landa-Ramírez, E., \& de Jesús Arredondo-Pantaleón, A. (2014). Herramienta PICO para la formulación y búsqueda de preguntas clínicamente relevantes en la psicooncología basada en la evidencia. Psicooncología, 11(2/3), 259.

Marins, R. B., Cecagno, S., Gonçalves, K. D., Braga, L. R., Ribeiro, J. P., \& Soares, M. C. (2020). Care techniques for pain relief in birthing. Revista de Pesquisa Cuidado é Fundamental Online, 276-281. https://doi.org/10.9789/2175-5361.rpcfo.v12.8502

Nagahama, E. E. I., \& Santiago, S. M. (2005). A institucionalização médica do parto no Brasil. Ciência \& Saúde Coletiva, $10,651-657$. 
Research, Society and Development, v. 10, n. 5, e53210515394, 2021

(CC BY 4.0) | ISSN 2525-3409 | DOI: http://dx.doi.org/10.33448/rsd-v10i5.15394

Pereira, P. S. L., Gomes, I. S., Ribeiro, Í. A. P., Morais, J. D. C., Gouveia, M. T. D. O., Nascimento, M. V. F., \& Sales, I. M. M. (2018). Tecnologias não invasivas de cuidado: percepção das puérperas. Rev. enferm. UFPE on line, 2129-2136.

Pereira, S. B., Diaz, C. M. G., Backes, M. T. S., Ferreira, C. L. D. L., \& Backes, D. S. (2018). Boas práticas de atenção ao parto e ao nascimento na perspectiva de profissionais de saúde. Revista Brasileira de Enfermagem, 71, 1313-1319.

Segura, O. M. S (1996). Assistência ao Parto Normal: um guia prático. Brasília: Ministério da Saúde.

Silva, A. L. S., Nascimento, E. R. D., \& Coelho, E. D. A. C. (2015). Práticas de enfermeiras para promoção da dignificação, participação e autonomia de mulheres no parto normal. Escola Anna Nery, 19(3), 424-431

Soares, Y. K. D. C., Melo, S. S., Guimarães, T. M. M., Feitosa, V. C., \& Gouveia, M. T. D. O. (2017). Satisfação das puérperas atendidas em um centro de parto normal. Rev. enferm. UFPE on line, 4563-4573.

Stark, MA, Remynse, M., \& Zwelling, E. (2016). Importância do Ambiente de Parto para Apoiar o Parto Fisiológico. Journal of Obstetric, Gynecologic \& Neonatal Nursing, 45 (2), 285-294. https://doi.org/10.1016/j.jogn.2015.12.008

Vendrúscolo, C. T., \& Kruel, C. S. (2015). A história do parto: do domicílio ao hospital; das parteiras ao médico; de sujeito a objeto. Disciplinarum Sciential Ciências Humanas, 16(1), 95-107.

Zielinski, R. E., Brody, M. G., \& Low, L. K. (2016). O valor da equipe de maternidade na promoção do parto fisiológico. Journal of Obstetric, Gynecologic \& Neonatal Nursing, 45 (2), 276-284. https://doi.org/10.1016/j.jogn.2015.12.009 